\title{
Unraveling the not-so-large trion binding energy in monolayer black phosphorus
}

\author{
Deilmann, Thorsten; Thygesen, Kristian Sommer
}

Published in:

2D materials

Link to article, DOI:

10.1088/2053-1583/aadc28

Publication date:

2018

Document Version

Peer reviewed version

Link back to DTU Orbit

Citation (APA):

Deilmann, T., \& Thygesen, K. S. (2018). Unraveling the not-so-large trion binding energy in monolayer black phosphorus. 2D materials, 5(4), [041007]. https://doi.org/10.1088/2053-1583/aadc28

\section{General rights}

Copyright and moral rights for the publications made accessible in the public portal are retained by the authors and/or other copyright owners and it is a condition of accessing publications that users recognise and abide by the legal requirements associated with these rights.

- Users may download and print one copy of any publication from the public portal for the purpose of private study or research.

- You may not further distribute the material or use it for any profit-making activity or commercial gain

- You may freely distribute the URL identifying the publication in the public portal

If you believe that this document breaches copyright please contact us providing details, and we will remove access to the work immediately and investigate your claim. 


\title{
Unraveling the not-so-large trion binding energy in monolayer black phosphorus
}

\author{
Thorsten Deilmann ${ }^{*}$ \\ CAMD, Department of Physics, Technical University of Denmark, DK-2800 Kongens Lyngby, Denmark \\ Kristian Sommer Thygesen \\ CAMD, Department of Physics, Technical University of Denmark, DK-2800 Kongens Lyngby, Denmark and \\ Center for Nanostructured Graphene (CNG), Technical University of Denmark, DK-2800 Kongens Lyngby, Denmark
}

(Dated: August 14, 2018)

\begin{abstract}
Black phosphorus (bP) is a promising two-dimensional (2D) material for opto-electronic applications. Strongly bound excitons with binding energies up to $0.3 \mathrm{eV}$ and remarkably large trion binding energies up to $100 \mathrm{meV}$ have been observed for supported monolayer bP. Surprisingly, this trion binding energy is significantly larger than those found in other $2 \mathrm{D}$ materials (e.g. about $30 \mathrm{meV}$ in transition metal dichalcogenides). This has previously been ascribed to the quasi-1D nature of bP. In this work we show, using first principles calculations, that the trion binding energy of $\mathrm{bP}$ is indeed large $(80 \mathrm{meV})$ when referenced to the lowest bright exciton but only $30 \mathrm{meV}$ when its energy is measured relative to the lowest dark exciton. Our analysis thus shows that the trion binding energy in bP is not larger than in other $2 \mathrm{D}$ materials, and the previous conclusions have to be understood incorporating the large splitting between the dark and bright excitons in bP. We also explore the effect of substrate and in-plane strain of the exciton and trion binding energies and show that these effects do not change the main conclusions. Our results correct the misconception that trion binding energies in monolayer bP are particularly large due to its quasi-1D structure and contribute to the establishment of more a detailed understanding of optical properties of atomically thin semiconductors.
\end{abstract}

\section{Introduction}

While black phosphorus (bP) has been known in its bulk form for years the strong interest in 2D materials has lead to the mechanical exfoliation and intensive research on thin films down to monolayers [1-11. In particular bP is highly anisotropic and exhibits several interesting properties such as a band gap in the visible spectrum and a large hole-mobility which leads to good transport properties. As for other 2D semiconductors, the optical properties of few-layer bP are dominated by localized electronic excitations, most notably excitons (neutral electron-hole pairs) and charged trions (positive e-h-h or negative ee-h complexes). Overall, these properties make bP very interesting for opto-electronic devices.

Recently, Yang et al. have reported surprisingly large trion binding energies of $\sim 100 \mathrm{meV}$ in monolayer bP on $\mathrm{SiO}_{2} / \mathrm{Si}$ substrate [8] even though the measured exciton binding energies of $0.3 \mathrm{eV}$ are not particularly large (compared to other 2D semiconductors with similar band gaps [12]). In $2016 \mathrm{Xu}$ et al. claimed even larger trion binding energies of $\sim 160 \mathrm{meV}$ in few-layer bP at room temperature and justified them by the "quasi-1D nature" of bP [13. Using Wannier-Mott theory Chaves et al. have found smaller trion binding energies of up to $50 \mathrm{meV}$ in the monolayer bP [14] which is still a factor of two larger than those of transition metal dichalcogenides (TMDCs) [15. At first sight, the explanation that the quasi-1D structure of bP is responsible for the huge trion binding energies appear plausible. Indeed, in other 1D systems like carbon nanotubes and graphene nanoribbons trion

\footnotetext{
* thorsten.deilmann@wwu.de
}

binding energies of more than $100 \mathrm{meV}$ have been measured and predicted [16 20]. However, the theoretical studies have shown that large trion binding energies come along with large exciton binding as both result from the enhanced Coulomb interaction in these low dimensional materials $18-20$.

To resolve the discrepancy between large trion binding energies and moderate exciton binding energies in the monolayer bP, we employ ab initio calculations treating trions and excitons on an equal footing 2, 21 23. We find that trions in bP have similar anisotropic properties as the excitons and their binding energies are only about $30 \mathrm{meV}$ below the lowest (triplet) exciton. Their binding energies are thus similar to other 2D materials, while the energetic separation of the bright trion and the lowest bright (singlet) exciton is distinctly larger (about $80 \mathrm{meV}$ ) explaining the previously reported extraordinary binding energies. Finally, we show that substrate screening and in-plane strain (which might be present in experiment) only slightly modify the binding energies but do not qualitatively change our conclusions.

\section{Free-standing monolayer black phosphorus}

Black phosphorus is a highly anisotropic material. As discussed in many previous works (e.g. Ref. 2]) it forms 1D-like chains which we align to the $y$ axis in this work. This results in highly anisotropic electronic properties as evident from the band structure in Fig. 1 (see method section for further details). In particular this anisotropy is clearly visible close to the direct gap at $\Gamma$. While for $\Gamma \rightarrow \mathrm{Y}$ a parabolic dispersion is found, the $\Gamma \rightarrow \mathrm{X}$ direction is distinctly more flat. We calculate effective masses of $0.2 / 4.3 m_{0}$ for the holes in the valence band along $k_{x} / k_{y}$ and $0.2 / 1.1 m_{0}$ for electrons in the conduction band (when applying the $G W$ approximation). To 


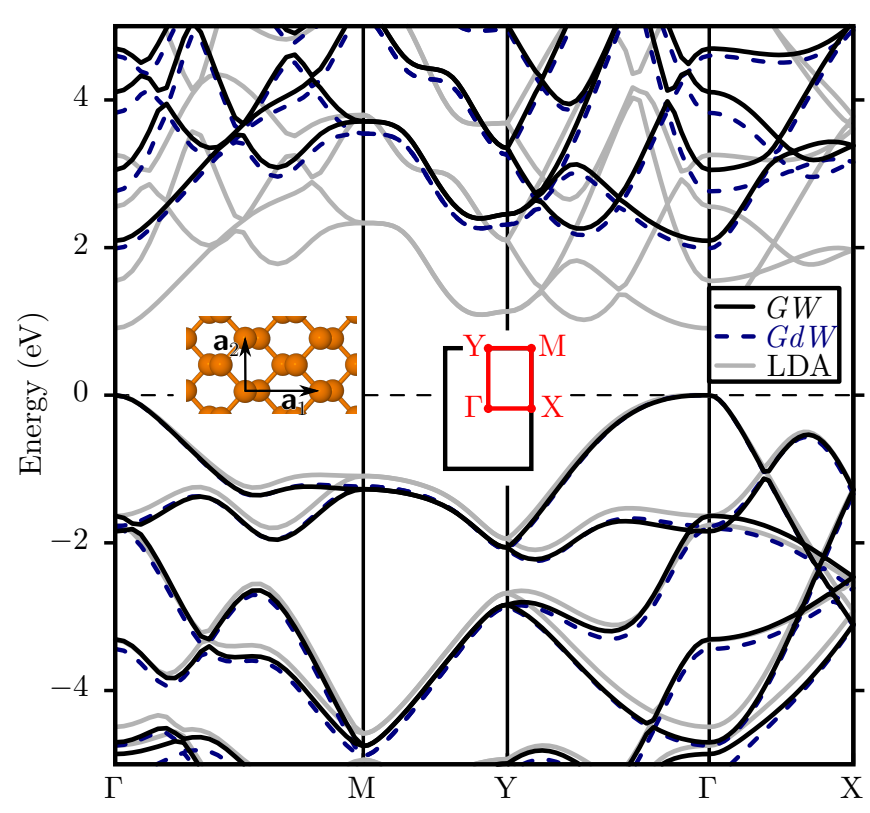

FIG. 1. Band structure employing the LDA (grey), in the $G d W$ approximation (dashed blue), and in the $G W$ approximation (black). In all calculations the valence band maximum has been set to zero. The insets show the a top view of the unit cell and the 2D Brillouin zone.

investigate the influence of the supporting surface we will employ the $G d W$ approximation [24] as well. Note that this approximation leads to nearly identical effective masses while the gap of $1.99 \mathrm{eV}$ is slightly smaller than $2.09 \mathrm{eV}$ using the $G W$ approximation (LDA: $0.91 \mathrm{eV}$ ). Overall band structures in both approaches $(G d W$ and $G W)$ are in good agreement and compare well with previous studies [1, 2]. Previous calculations for the direct gap at $\Gamma$ ranges from $2.03 \mathrm{eV}[25$ to $2.31 \mathrm{eV}[6]$, in decent agreement with experimental results of $2.2 \mathrm{eV}$ [7].

In the next step we will focus on the asymmetric optical properties which we evaluate the optical properties by solving the Bethe-Salpeter equation [26, 27, in the Tamm-Dancoff approximation. This leads to the diagonalization of the Hamiltonian

$$
\hat{H}_{\mathbf{v} \mathbf{c}, \mathbf{v}^{\prime} \mathbf{c}^{\prime}}^{(e h)}=\left(\epsilon_{\mathbf{c}}-\epsilon_{\mathbf{v}}\right) \delta_{\mathbf{c c}^{\prime}} \delta_{\mathbf{v v}^{\prime}}-\left(W_{\mathbf{v}^{\prime} \mathbf{c}, \mathbf{v} \mathbf{v}^{\prime}}-V_{\mathbf{v}^{\prime} \mathbf{c}, \mathbf{c}^{\prime} \mathbf{v}}\right)
$$

in the electron-hole pair basis with $\mathbf{v}=\left(v, \mathbf{k}_{v}\right)$ denoting the band and $\mathbf{k}$ index of the hole, respectively. This Hamiltonian consists of the difference of the quasiparticle energies $\left(\epsilon_{\mathbf{c}}-\epsilon_{\mathbf{v}}\right)$ as well as the screened/bare Coulomb interaction $(W / V)$ and may lead to bound electronic excitations below the band gap. The positively and negatively charged trions (electron-hole-hole or electron-electron-hole) are described by our recently developed methodology [19], which has been successfully applied to carbon nanotubes as well as 2D materials [19, 28]. E.g. the matrix elements of the eeh Hamil-

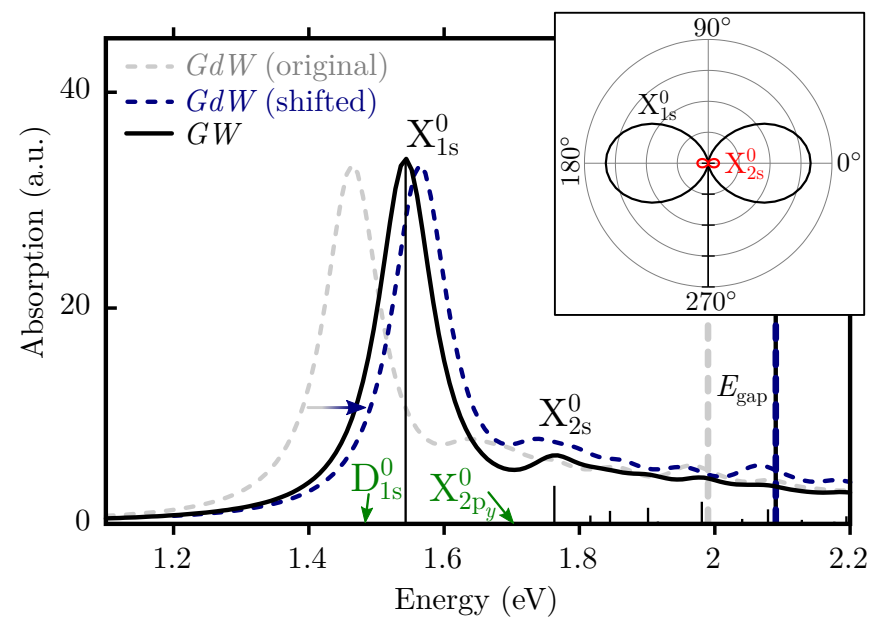

FIG. 2. Optical absorption spectrum of excitons with polarization in the $x$ direction. The black curve shows the results of the BSE based on $G W$ while the dashed grey shows the $G d W+$ BSE result (both calculations employ a $30 \times 42$ mesh). In addition the $G d W$ spectrum is shifted upwards by $0.1 \mathrm{eV}$ to match the $G W$ band gap (blue curve). A Lorentzian broadening of $50 \mathrm{meV}$ is applied. In the inset we show the polarization of the two lowest optical bright excitons $\mathrm{X}_{1 \mathrm{~s}}^{0}$ and $\mathrm{X}_{2 \mathrm{~s}}^{0}$.

tonian are given by

$$
\begin{aligned}
\hat{H}_{\mathbf{v} \mathbf{c}_{1} \mathbf{c}_{2}, \mathbf{v}^{\prime} \mathbf{c}_{1}^{\prime} \mathbf{c}_{2}^{\prime}}^{(e h)} & \left(\epsilon_{\mathbf{c}_{1}}+\epsilon_{\mathbf{c}_{2}}-\epsilon_{\mathbf{v}}\right) \delta_{\mathbf{c}_{1} \mathbf{c}_{1}^{\prime}} \delta_{\mathbf{c}_{2} \mathbf{c}_{2}^{\prime}} \delta_{\mathbf{v v}^{\prime}} \\
& +\left(W_{\mathbf{c}_{1} \mathbf{c}_{2}, \mathbf{c}_{1}^{\prime} \mathbf{c}_{2}^{\prime}}-W_{\mathbf{c}_{1} \mathbf{c}_{2}, \mathbf{c}_{2}^{\prime} \mathbf{c}_{1}^{\prime}}\right) \delta_{\mathbf{v} \mathbf{v}^{\prime}} \\
& -\left(W_{\mathbf{v}^{\prime} \mathbf{c}_{1}, \mathbf{v c}_{1}^{\prime}}-V_{\mathbf{v}^{\prime} \mathbf{c}_{1}, \mathbf{c}_{1}^{\prime} \mathbf{v}}\right) \delta_{\mathbf{c}_{2} \mathbf{c}_{2}^{\prime}} \\
& -\left(W_{\mathbf{v}^{\prime} \mathbf{c}_{2}, \mathbf{v} \mathbf{c}_{2}^{\prime}}-V_{\mathbf{v}^{\prime} \mathbf{c}_{2}, \mathbf{c}_{2}^{\prime} \mathbf{v}}\right) \delta_{\mathbf{c}_{1} \mathbf{c}_{1}^{\prime}}
\end{aligned}
$$

To provide a reliable description of the optical properties we have converged the employed meshes (for the band structure, self energy, and the electron-hole interaction) as well as the number of used valence and conduction bands.

Having solved the BSE (1) and trion Hamiltonian (2) we can compare the optical absorption spectra of neutral excitations employing the $G W$ and $G d W$ approximation in Fig. 2, The lowest excitations arise from transitions between the highest valence and lowest conduction band close to $\Gamma$. The first two optical bright states have 1s $\left(\mathrm{X}_{1 \mathrm{~s}}^{0}\right)$ and $2 \mathrm{~s}\left(\mathrm{X}_{2 \mathrm{~s}}^{0}\right)$ character and their energies with respect to the ground state are found at 1.54 and $1.76 \mathrm{eV}$. We note that our results include the dark $\mathrm{X}_{2 \mathrm{p}_{y}}^{0}$ in between these states, which is shifted below the $2 s$ state due to the inhomogeneous screening [29] and the asymmetry as discussed in Ref. 30. In comparison to the $G W+\mathrm{BSE}$ approach (black) the optical spectrum employing $G d W+\mathrm{BSE}$ is shifted to lower energies by about $0.1 \mathrm{eV}$ which is a consequence of the reduced gap. To compare the relative alignment of the $G d W$ and $G W$ approximation we employ a rigid scissors shift to match the gap (blue dashed line). Both spectra are in excellent agreement, both in terms of spectral positions and optical weights. 
While the spectra in Fig. 2 are shown for light polarization in the $x$ direction, the inset reveals the polarization with respect to the $\Gamma \mathrm{X}$ (arm-chair) direction. For both low-energy states $\mathrm{X}_{1 \mathrm{~s}}^{0}$ and $\mathrm{X}_{2 \mathrm{~s}}^{0}$ we observe a distinct asymmetric behaviour. We note that the first optically bright transition in for light polarized in the $y$ direction is far above the band gap (see e.g. Ref. 2]). Therefore we focus on light polarization in $x$ in this study.

Beside the neutral excitons we consider positively and negatively charged trions which have been much less discussed theoretically and mostly based on models. Employing our ab initio derived Hamiltonian (2) we find strongly bound trions [Fig. 3(a)]. The lowest trion state $\mathrm{X}_{1 \mathrm{~s}}^{+}$is found at $80 \mathrm{meV}$ below the corresponding $\mathrm{X}_{1 \mathrm{~s}}^{0}$ exciton which is typically defined as the trion binding energy

$$
E_{\mathrm{b}}^{\operatorname{Tr}^{+}}=E_{\mathrm{X}_{1 \mathrm{~s}}^{+}}-E_{\mathrm{X}_{1 \mathrm{~s}}^{0}}
$$

A detailed discussion of the splitting of different peaks can be found at the end of this section. We note in passing that we find several additional resonant trions and trions corresponding to higher lying excitons [20. If we assume a negative doping, very similar result are observed. We find the trion binding energy of positive and negative species to deviate only by about $1 \mathrm{meV}$ (negatively charged trions are lower in energy) which is at the limit of our the accuracy.

Employing Wannier-Mott theory Chaves et al. 14. have observed qualitatively similar results. Their calculations show excitons (with binding energies of $0.74 \mathrm{eV}$ ) and trions (with binding energies of $52 \mathrm{meV}$ ) below the quasi-particle gap of $2.49 \mathrm{eV}$. While we find a slightly smaller gap and exciton binding energy, our trion binding energy turns out to be slightly larger. We speculate that this may be related to the slightly different effective masses as well as to the employed model which does not capture the full $q$-dependency of the dielectric function.

While in other semiconducting 2D materials (like transition metal dichalcogenides [31]) the spin-orbit interaction is large, its influence is negligible for black phosphorus (and it is therefore excluded from our calculations). Hence the spin remains a good quantum number and for the exciton we observe four different possible spin states. This includes three optically dark triplet states $[|\uparrow \uparrow\rangle, 1 / \sqrt{2}(|\uparrow \downarrow\rangle+|\downarrow \uparrow\rangle)$, and $|\downarrow \downarrow\rangle]$ and one optically bright singlet state $[1 / \sqrt{2}(|\uparrow \downarrow\rangle-|\downarrow \uparrow\rangle)][26$. For black phosphorus we find the triplet states $50 \mathrm{meV}$ lower than the singlet state. In comparison to excitons, the combination of the three different spins and splitting of charged trions is slightly more complicated. Mixing the triplet state with a further particle results in a quartet $\left|S=\frac{3}{2}\right\rangle$ and a doublet $\left|S=\frac{1}{2}\right\rangle$. A further doublet $\left|S=\frac{1}{2}\right\rangle$ stems from the mixing of the additional spin with the previous singlet state. For energetically low-lying states in bP we find that the latter doublet is optically active while the six other states are dark. While the dark quartet is slightly lower in energy we find the doublets only marginally split in energy. The resulting excitation spectrum is sketched
TABLE I. Shift of the quasi-particle band gap, the excitation energies and corresponding binding energies due to a $\mathrm{SiO}_{2}$ substrate in meV. The results are evaluated using the $G d W$ approximation.

$$
\begin{array}{ccccc}
E_{\text {gap }} & E_{1 \mathrm{~s}}^{\mathrm{X}^{0}} & E_{\mathrm{b}}^{\mathrm{X}_{1 \mathrm{~s}}^{0}} & E_{1 \mathrm{~s}}^{\mathrm{X}^{+}} & E_{\mathrm{b}}^{\mathrm{X}_{1 \mathrm{~s}}^{+}} \\
\hline-160 & -60 & -100 & -50 & -10
\end{array}
$$

in Fig. 3(c). We note that exactly at $\Gamma$ only one bright doublet can be realized due to the Pauli exclusion principle. Furthermore we emphasize that the bright trion (doublet) will split in a magnetic field in contrast to the bright excitons (singlet state),

Having discussed the different spin states, we come back to the discussion of the large trion binding energy. Eq. (3) uses the bright exciton $\mathrm{X}_{1 \mathrm{~s}}^{0}$ as reference (which is easily accessible experimentally), however the dark exciton $\mathrm{D}_{1 \mathrm{~s}}^{0}(|S=1\rangle)$ can also form a bright trion by binding an additional electron (hole) from the CBM (VBM). The trion binding energy with reference to $D_{1 \mathrm{~s}}^{0}$ is about $30 \mathrm{meV}$ and thus of similar similar size compared to other 2D material (e.g. TMDCs [15, 28, 32]). Therefore we trace back the large trion binding energy to the spin structure in contrast to previous studies arguing for the "quasi-1D" nature of bP [8, 13.

Strain and dielectric polarisation of the substrate Until now we have discussed the properties of the freestanding monolayer. However, such a perfect undisturbed system is hard to achieve experimentally. Monolayers are typically placed on the substrate, e.g. $\mathrm{SiO}_{2}$ [8]. This changes their dielectric environment and will thus influence the electronic and optical properties. Furthermore the monolayer properties may be modified by strain, which might stem from alignment to the substrate.

To account for the dielectric screening by the surface we apply the $G d W$ method [24]. The substrate is simulated by 6 layers of $\mathrm{SiO}_{2}$ in a distance of $3 \AA$ from the black phosphorus. As discussed previously (for details of this procedure see Refs. 28, 33.) we only include the dielectric response of the substrate. The resulting differences of $\mathrm{bP}$ in vacuum and above the surface are shown in Tab. I. As observed for other 2D material [28, we find a large renormalization of the gap as well as a decrease of the exciton binding energy due to the additional screening by the substrate. However, these effects do not perfectly cancel and a small red-shift of $60 \mathrm{meV}$ remains for $\mathrm{X}_{1 \mathrm{~s}}^{0}$. Similar as the neutral exciton also the $\mathrm{X}_{1 \mathrm{~s}}^{+}$trion is red-shifted. These shifts result in a slightly reduced trion binding energy. In Ref. [14 Chaves et al. modelled the effects of substrates (dielectric constant $\epsilon_{2}=3.8$ ). They observe a slightly larger decrease of exciton and trion binding energy. However their change of $17 \mathrm{meV}$ is in reasonable agreement with the results of the ab initio calculation. We note that the energetically lowest dark exciton changes in a similar way and within our numerical accuracy we find the same results as shown in Tab. I. 
(a)

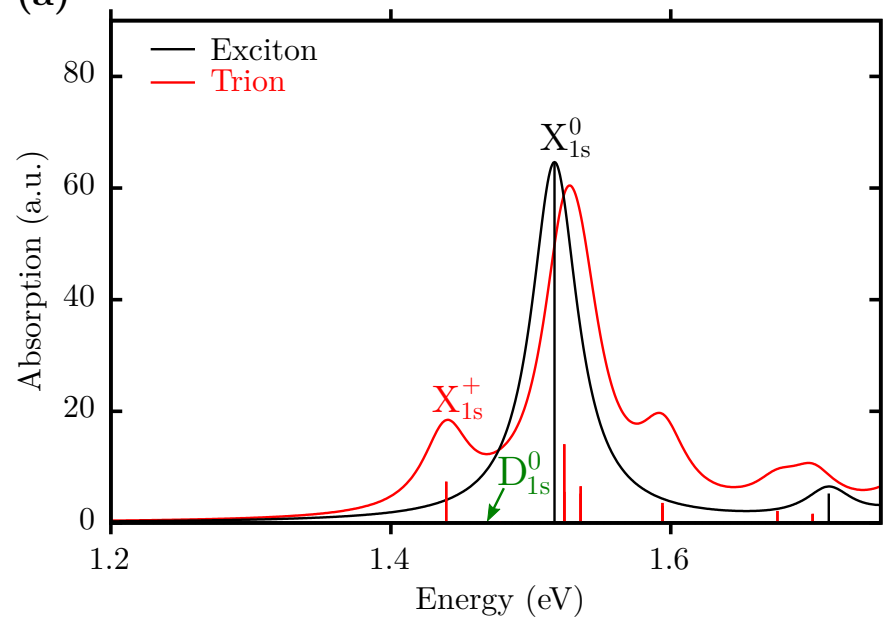

(b)

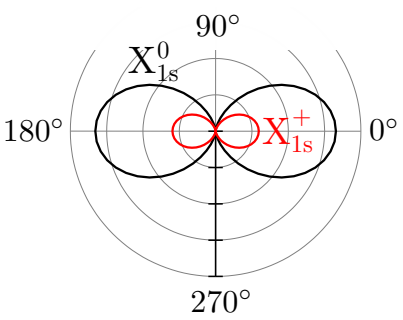

(c)

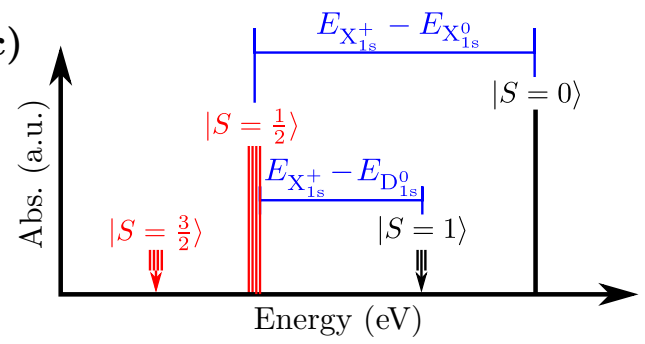

FIG. 3. (a) Optical absorption spectrum of neutral excitons (black) and positively charged trions (red) employing a $15 \times 21 \times 1$ mesh. We note that we cannot determine the relative weight of excitons and trions, which are shown in the limit of vanishing doping. A Lorentzian broadening of $20 \mathrm{meV}$ is applied. (b) Polarization of the lowest bright trion $\mathrm{X}_{1 \mathrm{~s}}^{+}$compared to the $\mathrm{X}_{1 \mathrm{~s}}^{0}$ exciton. (c) Sketch of the energetic ordering including bright (singlet $|S=0\rangle$ excitons and doublet $\left|S=\frac{1}{2}\right\rangle$ trions) and dark excitations (triplet $|S=1\rangle$ excitons and quartet $\left|S=\frac{3}{2}\right\rangle$ trions). In blue the differences to the singlet and triplet states are indicated. We note that at $\Gamma$ no $\left|S=\frac{3}{2}\right\rangle$ trions can be formed.
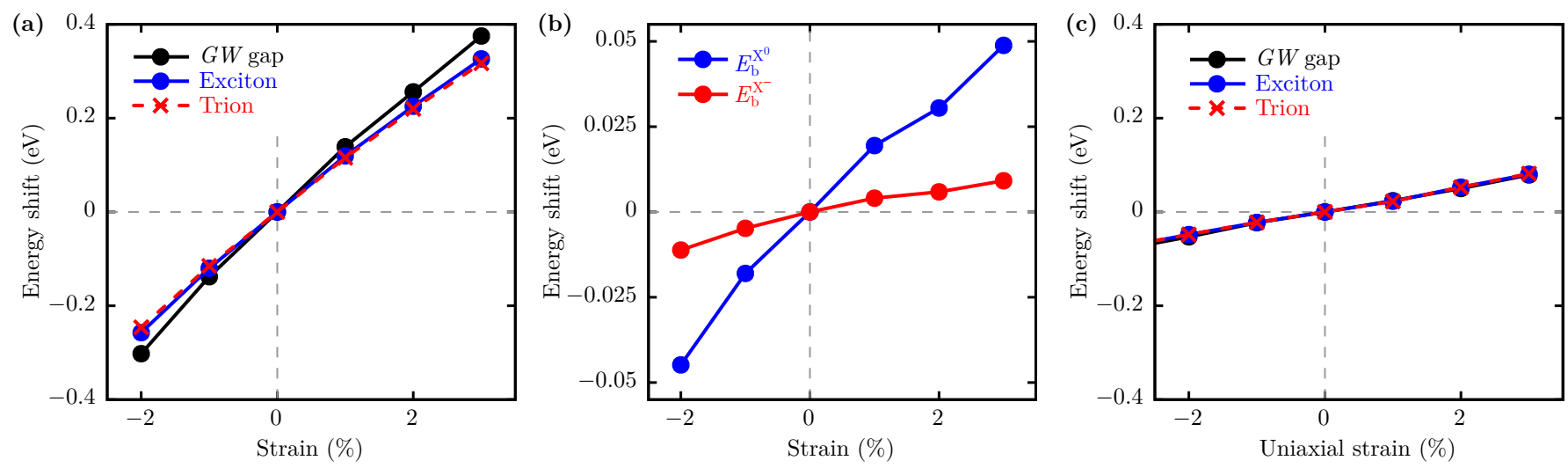

FIG. 4. (a) Band gap, exciton and trion energy of black phosphorus as function of the applied biaxial strain. The corresponding quasi-particle calculations are evaluated employing the $G W$ approximation. (b) Energy shift of exciton and trion binding energy [differences of curves in (a)] with respect to the applied biaxial strain. (c) Band gap, exciton and trion energy for as function of the applied uniaxial strain (along the chain axis). The lines are shown as guide to the eye.

Next, we apply biaxial strain to the bP monolayer (as previously discussed, e.g. in Ref. [6]). While fixing the unit cell all atomic positions are relaxed. The energy shift of the band gap, the exciton and trion energy (of the lowest bright state) are shown in Fig. 4(a). All three quantities change nearly linearly for small applied strains. However, the different gradients result in modified exciton and trion binding energies [Fig. 4(b)]. For example stretching the material by $2 \%$ leads to a blue-shifted exciton and trion peaks by about $0.2 \mathrm{eV}$ while the exciton (trion) binding energy $E_{\mathrm{b}}^{\mathrm{X}^{0}}\left(E_{\mathrm{b}}^{\mathrm{X}^{0}}\right)$ is only increased by about $30 \mathrm{meV}(10 \mathrm{meV})$.

Furthermore it is instructive to investigate these quantities applying uniaxial strain in the strongly asymmet- ric monolayer. E.g. Du et al. have shown that uniaxial strain along the chain axis leads to negative Poisson ratio in monolayer bP [34. The energy shifts of the electronic and optical properties (Fig. 4(c)) show two interesting characteristics: (i) The energy shift is distinctly reduced compared to biaxial strain and (ii) the change of band gap and excitation energies are practically identical, i.e. the exciton/trion binding energy is unchanged (within our numerical accuracy) in contrast to biaxial strain. We note that the different behaviour of uniaxial and biaxial strain is already observed in our DFT calculations (DFT band gap). Here we find that the band gap increases when increasing $\mathbf{a}_{1}$ or $\mathbf{a}_{2}$, respectively. Thus for uniaxial strain the increase of the band gap when increasing 
the lattice in one direction is largely compensated by the decrease in the second direction while they add up for biaxial strain.

In comparison to the experimental trion binding energy of $\sim 100 \mathrm{meV}$ on a $\mathrm{SiO}_{2}$ substrate $[$ ] our calculated trion binding energy is lower. On the substrate we find about $70 \mathrm{meV}$ which might be slightly increased due to strain. As also concluded by Chaves et al. 14] we believe that the experimental value is only an upper bound and needs to be validated or corrected in the limit of vanishing doping. In contrast to Chaves et al. our ab initio calculations predict the trion binding energy with respect to the bright singlet exciton to be distinctly higher than for other 2D materials [28, while it is of similar size comparing with reference to the dark triplet exciton.

In summary, our results resolve the discrepancy between large trion binding energies and moderate exciton binding energies in the monolayer black phosphorus. By using first principles methods we find a trion binding energy of $30 \mathrm{meV}$ with reference to the lowest (dark) exciton, which is of similar size compared to other $2 \mathrm{D}$ materials. Only when referenced to the lowest bright exciton a larger splitting of the exciton and trion of about $80 \mathrm{meV}$ is observed. Because monolayers are typically placed on a substrate in experiment, we investigate the influence due to its dielectric screening. We find a small red shift of the excitations and slightly reduced binding energies similar to those found in other 2D materials. By applying negative biaxial strain this red shift can be enhanced or even turned into a small blue shift for positive strains. Methods

To describe the optical properties of black phosphorus (bP) from first principles we apply a hierarchy of methods. The structure is determined by a DFT calculation employing GPAW [35. We have employed the generalized gradient approximation (PBE) [36], a cutoff of $800 \mathrm{eV}$ for the plane wave basis and a $14 \times 10$ mesh to sample the first Brillouin zone. Using a fixed vertical cell size of $30 \AA$ we have optimized the two-dimensional unit cell and its atoms until the stresses and forces are below $0.005 \mathrm{eV} / \AA$, respectively. We find the unit cell to be $3.306 \AA \times 4.629 \AA$ with a P-P distance of $2.22 \AA$. These results are in good agreement to previous findings of Liu et al. [1]. We note that these are only minor changes compared to the optimized bulk lattice vectors of $3.313 \AA \times 4.564 \AA \times 11.315 \AA$. When we apply strain we change the unit cell size and only optimize the atomic positions.

For the further calculations we apply a Gaussian basis set [37, 38] (we use $s, p, d$, and $s^{*}$ functions with decay constants between 0.16 and 1.87 (in a.u.) to describe the $\mathrm{P}$ atoms). The electronic properties are evaluated and compared between LDA, $G d W(\mathrm{LDA})$ 24, and the $G W$ approximation. The last two methods require a second, auxiliary basis set to represent the two-point quantities (dielectric function, screened Coulomb interaction, etc.). For $G W$ we employ a plane wave basis with a cutoff of 7 Ry which is able to describe the gap with an accuracy of less than $50 \mathrm{meV}$ compared to the result extrapolated to an infinite basis size $\left(N_{\mathrm{PW}} \rightarrow \infty\right)$. In $G d W$ the numerical requirements are reduced and a cutoff of $3 \mathrm{Ry}$ is sufficient. To account for vanishing interactions to the neighbouring layers we extrapolate the band gap to $z \rightarrow \infty$. For a detailed discussion of the convergence of excitons and trions employing this methods we referee to previous studies [28, 32].

\section{Acknowledgements}

T.D. acknowledges financial support from the Villum foundation. The Center for Nanostructured Graphene (CNG) is sponsored by the Danish Research Foundation, Project No. DNRF103. This project has received funding from the European Research Council (ERC) under the European Union's Horizon 2020 research and innovation programme (grant agreement No 773122, LIMA).
[1] Han Liu, Adam T. Neal, Zhen Zhu, Zhe Luo, Xianfan $\mathrm{Xu}$, David Tománek, and Peide D. Ye, "Phosphorene: An Unexplored 2D Semiconductor with a High Hole Mobility," ACS Nano 8, 4033-4041 (2014)

[2] Vy Tran, Ryan Soklaski, Yufeng Liang, and Li Yang, "Layer-controlled band gap and anisotropic excitons in few-layer black phosphorus," Physical Review B 89, 235319 (2014)

[3] Likai Li, Yijun Yu, Guo Jun Ye, Qingqin Ge, Xuedong Ou, Hua Wu, Donglai Feng, Xian Hui Chen, and Yuanbo Zhang, "Black phosphorus field-effect transistors," $\mathrm{Na}$ ture Nanotechnology 9, 372-377 (2014-05).

[4] Fengnian Xia, Han Wang, and Yichen Jia, "Rediscovering black phosphorus as an anisotropic layered material for optoelectronics and electronics," Nature Communications 5, ncomms5458 (2014)

[5] A. N. Rudenko and M. I. Katsnelson, "Quasiparticle band structure and tight-binding model for single- and bilayer black phosphorus," Physical Review B 89, 201408
(2014)

[6] Deniz Çakır, Hasan Sahin, and François M. Peeters, "Tuning of the electronic and optical properties of singlelayer black phosphorus by strain," Physical Review B 90, 205421 (2014)

[7] Xiaomu Wang, Aaron M. Jones, Kyle L. Seyler, Vy Tran, Yichen Jia, Huan Zhao, Han Wang, Li Yang, Xiaodong $\mathrm{Xu}$, and Fengnian Xia, "Highly anisotropic and robust excitons in monolayer black phosphorus," Nature Nanotechnology 10, 517 (2015).

[8] Jiong Yang, Renjing Xu, Jiajie Pei, Ye Win Myint, Fan Wang, Zhu Wang, Shuang Zhang, Zongfu Yu, and Yuerui $\mathrm{Lu}$, "Optical tuning of exciton and trion emissions in monolayer phosphorene," Light: Science \& Applications 4, e312 (2015)

[9] Jingsi Qiao, Xianghua Kong, Zhi-Xin Hu, Feng Yang, and Wei Ji, "High-mobility transport anisotropy and linear dichroism in few-layer black phosphorus," Nature Communications 5, ncomms5475 (2014) 
[10] A. S. Rodin, A. Carvalho, and A. H. Castro Neto, "Strain-Induced Gap Modification in Black Phosphorus," Physical Review Letters 112, 176801 (2014).

[11] A. Surrente, A. A. Mitioglu, K. Galkowski, W. Tabis, D. K. Maude, and P. Plochocka, "Excitons in atomically thin black phosphorus," Physical Review B 93, 121405 (2016)

[12] Andres Castellanos-Gomez, "Why all the fuss about 2D semiconductors?" Nature Photonics 10, 202204 (2016)

[13] Renjing $\mathrm{Xu}$, Shuang Zhang, Fan Wang, Jiong Yang, Zhu Wang, Jiajie Pei, Ye Win Myint, Bobin Xing, Zongfu $\mathrm{Yu}$, Lan Fu, Qinghua Qin, and Yuerui Lu, "Extraordinarily Bound Quasi-One-Dimensional Trions in Two-Dimensional Phosphorene Atomic Semiconductors," ACS Nano 10, 2046-2053 (2016).

[14] A. Chaves, M. Z. Mayers, F. M. Peeters, and D. R. Reichman, "Theoretical investigation of electron-hole complexes in anisotropic two-dimensional materials," Physical Review B 93, 115314 (2016).

[15] Tony Low, Andrey Chaves, Joshua D. Caldwell, Anshuman Kumar, Nicholas X. Fang, Phaedon Avouris, Tony F. Heinz, Francisco Guinea, Luis Martin-Moreno, and Frank Koppens, "Polaritons in layered twodimensional materials," Nature Materials 16, 182194 (2016)

[16] Ryusuke Matsunaga, Kazunari Matsuda, and Yoshihiko Kanemitsu, "Observation of Charged Excitons in HoleDoped Carbon Nanotubes Using Photoluminescence and Absorption Spectroscopy," Phys. Rev. Lett. 106, 037404 (2011)

[17] Silvia M. Santos, Bertrand Yuma, Stéphane Berciaud, Jonah Shaver, Mathieu Gallart, Pierre Gilliot, Laurent Cognet, and Brahim Lounis, "All-Optical Trion Generation in Single-Walled Carbon Nanotubes," Phys. Rev. Lett. 107, 187401 (2011).

[18] Troels F. Rønnow, Thomas G. Pedersen, and Horia D. Cornean, "Stability of singlet and triplet trions in carbon nanotubes," Physics Letters A 373, 1478 - 1481 (2009).

[19] Thorsten Deilmann, Matthias Drüppel, and Michael Rohlfing, "Three-particle correlation from a Many-Body Perspective: Trions in a Carbon Nanotube," Phys. Rev. Lett. 116, 196804 (2016).

[20] Thorsten Deilmann and Michael Rohlfing, "Huge trionic effects in graphene nanoribbons," Nano Letters (2017), 10.1021/acs.nanolett.7b03111.

[21] Pierluigi Cudazzo, Lorenzo Sponza, Christine Giorgetti, Lucia Reining, Francesco Sottile, and Matteo Gatti, "Exciton Band Structure in Two-Dimensional Materials," Physical Review Letters 116, 066803 (2016).

[22] F. Ferreira and R. M. Ribeiro, "Improvements in the \$GW $\$$ and Bethe-Salpeter-equation calculations on phosphorene," Physical Review B 96, 115431 (2017)

[23] Diana Y. Qiu, Felipe H. da Jornada, and Steven G. Louie, "Environmental Screening Effects in 2D Materials: Renormalization of the Bandgap, Electronic Structure, and Optical Spectra of Few-Layer Black Phosphorus," Nano Letters , 7b01365 (2017).

[24] Michael Rohlfing, "Electronic excitations from a perturbative LDA+GdW approach," Phys. Rev. B 82, 205127 (2010)

[25] Filip A. Rasmussen, Per S. Schmidt, Kirsten T. Winther, and Kristian S. Thygesen, "Efficient many-body calculations for two-dimensional materials using exact limits for the screened potential: Band gaps of $\mathrm{MoS}_{2}, \mathrm{~h}-\mathrm{bn}$, and phosphorene," Physical Review B 94, 155406 (2016).

[26] Michael Rohlfing and Steven G. Louie, "Electron-hole excitations and optical spectra from first principles," Phys. Rev. B 62, 4927-4944 (2000).

[27] Giovanni Onida, Lucia Reining, and Angel Rubio, "Electronic excitations: Density-functional versus many-body Green's-function approaches," Rev. Mod. Phys. 74, 601659 (2002)

[28] Matthias Drppel, Thorsten Deilmann, Peter Krüger, and Michael Rohlfing, "Diversity of trion states and substrate effects in the optical properties of an MoS2 monolayer," Nature Communications 8, 2117 (2017).

[29] Diana Y. Qiu, Felipe H. da Jornada, and Steven G. Louie, "Screening and Many-Body Effects in TwoDimensional Crystals: Monolayer $\mathrm{MoS}_{2}$," Phys. Rev. B 93, 235435 (2016).

[30] A. Chaves, Tony Low, P. Avouris, D. Çakır, and F. M. Peeters, "Anisotropic exciton Stark shift in black phosphorus," Physical Review B 91, 155311 (2015).

[31] Th. Böker, R. Severin, A. Müller, C. Janowitz, R. Manzke, D. Voß, P. Krüger, A. Mazur, and J. Pollmann, "Band structure of MoS2, MoSe2 , and $\alpha$ MoTe2 : Angle-resolved photoelectron spectroscopy and $a b$ initio calculations," Physical Review B 64 (2001), 10.1103/PhysRevB.64.235305

[32] Thorsten Deilmann and Kristian Sommer Thygesen, "Dark excitations in monolayer transition metal dichalcogenides," Phys. Rev. B 96, 201113 (2017).

[33] Taner Esat, Thorsten Deilmann, Benedikt Lechtenberg, Christian Wagner, Peter Krüger, Ruslan Temirov, Frithjof B. Anders, Michael Rohlfing, and F. Stefan Tautz, "Transfering spin into an extended pi orbital of a large molecule," Phys. Rev. B 91, 144415 (2015).

[34] Yuchen Du, Jesse Maassen, Wangran Wu, Zhe Luo, Xianfan $\mathrm{Xu}$, and Peide D. Ye, "Auxetic black phosphorus: A 2d material with negative poisson's ratio," Nano Letters 16, 6701-6708 (2016).

[35] J Enkovaara, C Rostgaard, J J Mortensen, J Chen, M Dułak, L Ferrighi, J Gavnholt, C Glinsvad, V Haikola, H A Hansen, H H Kristoffersen, M Kuisma, A H Larsen, L Lehtovaara, M Ljungberg, O Lopez-Acevedo, P G Moses, J Ojanen, T Olsen, V Petzold, N A Romero, J Stausholm-Møller, M Strange, G A Tritsaris, M Vanin, M Walter, B Hammer, H Häkkinen, G K H Madsen, R M Nieminen, J K Nørskov, M Puska, T T Rantala, J Schiøtz, K S Thygesen, and K W Jacobsen, "Electronic structure calculations with GPAW: A real-space implementation of the projector augmented-wave method," Journal of Physics: Condensed Matter 22, 253202 (2010)

[36] John P. Perdew, Kieron Burke, and Matthias Ernzerhof, "Generalized Gradient Approximation Made Simple," Phys. Rev. Lett. 77, 3865-3868 (1996)

[37] Jürgen Wieferink, Peter Krüger, and Johannes Pollmann, "Improved hybrid algorithm with Gaussian basis sets and plane waves: First-principles calculations of ethylene adsorption on $\beta$-SiC(001)- $(3 \times 2)$," Physical Review B 74, 205311 (2006)

[38] Thorsten Deilmann, Peter Krüger, Michael Rohlfing, and Daniel Wegner, "Adsorption and STM imaging of tetracyanoethylene on $\mathrm{Ag}(001)$ : An ab initio study," Phys. Rev. B 89, 045405 (2014). 